\title{
Fortalezas costeras del Oeste y Norte de Marruecos (S.XV- S.XVII)
}

\section{Faissal Cherradi Akbil}

Ministerio de Cultura del Reino de Marruecos. Rabat, Marruecos, cherradifaissal@yahoo.es

\begin{abstract}
I have the intention to speak about military architecture developed during Portuguese occupation between 1415, the conquest of Ceuta, and 1769, The Saudi Dynasty conquered the city of Mazagan (Currently named El Jadida).

The Portugueses started conquering the harbor of Ceuta because in that moment was the key of the commerce between Africa and Europe. They built a lot of strategic places.

During 300 years, Portugueses were fighting against Morocco Army. Currently, we have a lot of buildings set around Atlantic and Mediterranean coasts as the City of Mazagan (El Jadida), Azemmour o Mogador (Essaouira).

Currently, we have got common heritage shared in modern ages. This heritage was recognized by the Moroccan Government and International Communities and it was declared as World Heritage bt UNESCO. Even though we have encouraged the heritage to refurb and rehab it, we really know we have a lot of work to do yet.
\end{abstract}

Keywords: fortification, transformations, military architecture

\section{Introducción}

La herencia portuguesa en África del Norte nos permite trazar un periodo significativo de la historia de las fortificaciones. Nos permite apreciar las grandes transformaciones morfológicas que han caracterizado la geometría de las fortificaciones durante el paso entre el periodo de la Edad Media y el periodo del Renacimiento. La conquista portuguesa, comenzó con la toma de Ceuta y continúo con los grandes descubrimientos marítimos del siglo XVI y XVII, integrando dos innovaciones tecnológicas cruciales, como fueron la mejora de la navegación y la construcción de fuertes bastionados.

Mientras que la primera permitió a las carabelas y a los navíos portugueses desplazarse con mucha más facilidad y agilidad, la segunda permitió la construcción de instalaciones prácticamente inexpugnables.

Las nuevas técnicas militares de defensa se desarrollaron y fueron probadas en África del Norte que fue su primera colonia.

Durante ese periodo de desarrollo de las técnicas militares en el que se pasó de la defensa de las armas blancas a la utilización masiva de las armas de fuego especialmente cañones. Las construcciones tuvieron que asumir el cambio pasando de edificios con torres cuadradas y muros rectos a otros con torres más complejas incluso circulares, protobastiones para llegar posteriormente a la defensa con frentes bastionados y baluartes. Dependiendo así mismo de la orografía del terreno, los recursos financieros y las necesidades inmediatas. 
Concerniente a la forma geométrica exterior de las torres, se abandonan definitivamente las torres rectangulares o cuadradas por el uso de las torres circulares. Esta transformación está ligada al empleo de armas de fuego que lanzaban proyectiles más potentes y más rápidos.

La fuerza de impacto de un proyectil es proporcional al ángulo de impacto, siendo los 90 grados donde se obtiene la máxima capacidad de destrucción, cambiando a las torres redondas donde solo existe una línea ortogonal al plano de tiro.

También por la necesidad de albergar la artillería hizo que se incrementara la superficie, las dimensiones y la estructura de las torres ya que se necesitaba espacio para la maniobra de los cañones así que para su retroceso en el momento del disparo y poder soportar las fuertes vibraciones que estos producían. Igualmente las torres debían estar lo suficientemente ventiladas a causa del humo que provocaban los cañones en el momento de los disparos.

Repercutiendo la utilización de las armas de fuego en la dimensión de las aperturas con nuevas tipologías de troneras, barbacanas, saeteras, etc.

Dentro de la estrategia defensiva, las murallas también se transformaron morfológicamente y se adaptaron al tipo de defensa, realizándolas más anchas, más bajas y reforzadas. Para proteger los bajos de los muros que eran la parte más vulnerable construían unos salientes.

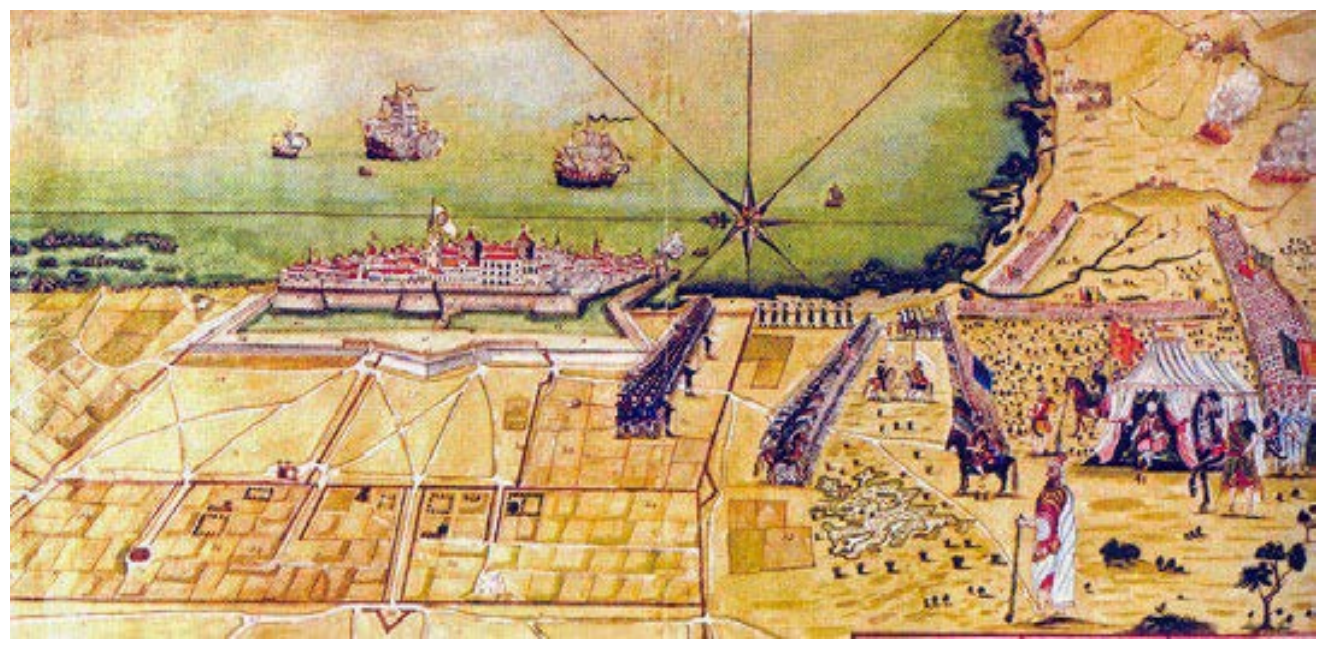

Fig. 1- Mazagan.

\section{Descripción histórica}

Los portugueses se instalaron por primera vez sobre el sitio de Mazagan en el año 1502. Había una sola construcción sobre este sitio que consistía en una torre llamada El-Bija. Después de unos años de ocupación en el que solo había construcciones temporales, los portugueses decidieron construir una ciudadela en el año 1514 concebida por los hermanos Francisco y Diego de Arruda que trabajaron también sobre otras fortificaciones y medinas marroquíes. En el año 1541, después de la perdida de la ciudad de
Agadir, los portugueses decidieron agrandar la ciudadela y construir fortificaciones complementarias. La concepción fue confiada a un equipo de ingenieros y de arquitectos compuesto por Joao Ribeiro y del español Juan Castillo y del italiano, Benedetto de Ravenna. De 1541 a 1548, el gobernador de la fortaleza era Louis de Loureiro, que ya había ejercido esta misma función en Ceuta y Mogador. En esa época, la ciudad de Mazagan conoció un desarrollo rápido, con la construcción de conjuntos religiosos, de una gran importancia en esa época de confrontaciones religiosas. A fines 
del siglo XVI, la fortificación albergaba cuatro iglesias y varias capillas.

Después de dos siglos y medio de ocupación, el periodo lusitano de Mazagan, ultima de las fortalezas portuguesas en Marruecos, llego a su fin en 1769. Después de la firma del tratado de paz con el Sultan Sidi Mohammed Ben Abdellah (1757-1790), los portugueses abandonaron precipitadamente la plaza por la puerta del Mar sin poder llevarse sus bienes, minando la entrada principal para que explotara cuando los marroquíes forzaran la entrada.
Explosiones que hicieron un gran número de victimas así como la destrucción del bastión del Gobernador y de una gran parte de la muralla. La ciudad quedo abandonada durante más de medio siglo, a la que se le otorgo el nombre de "la Destruida". Hasta que a mediados del siglo XIX, el Sultan Moulay Abderrahman ordenó al pacha de la región realizar el levantamiento de las partes destruidas su restauración así como la nueva construcción de una mezquita. Prohibió la utilización del nombre Mazagan, renombrando la ciudad con el nombre de“Al-Jadida”, "la Nueva”.

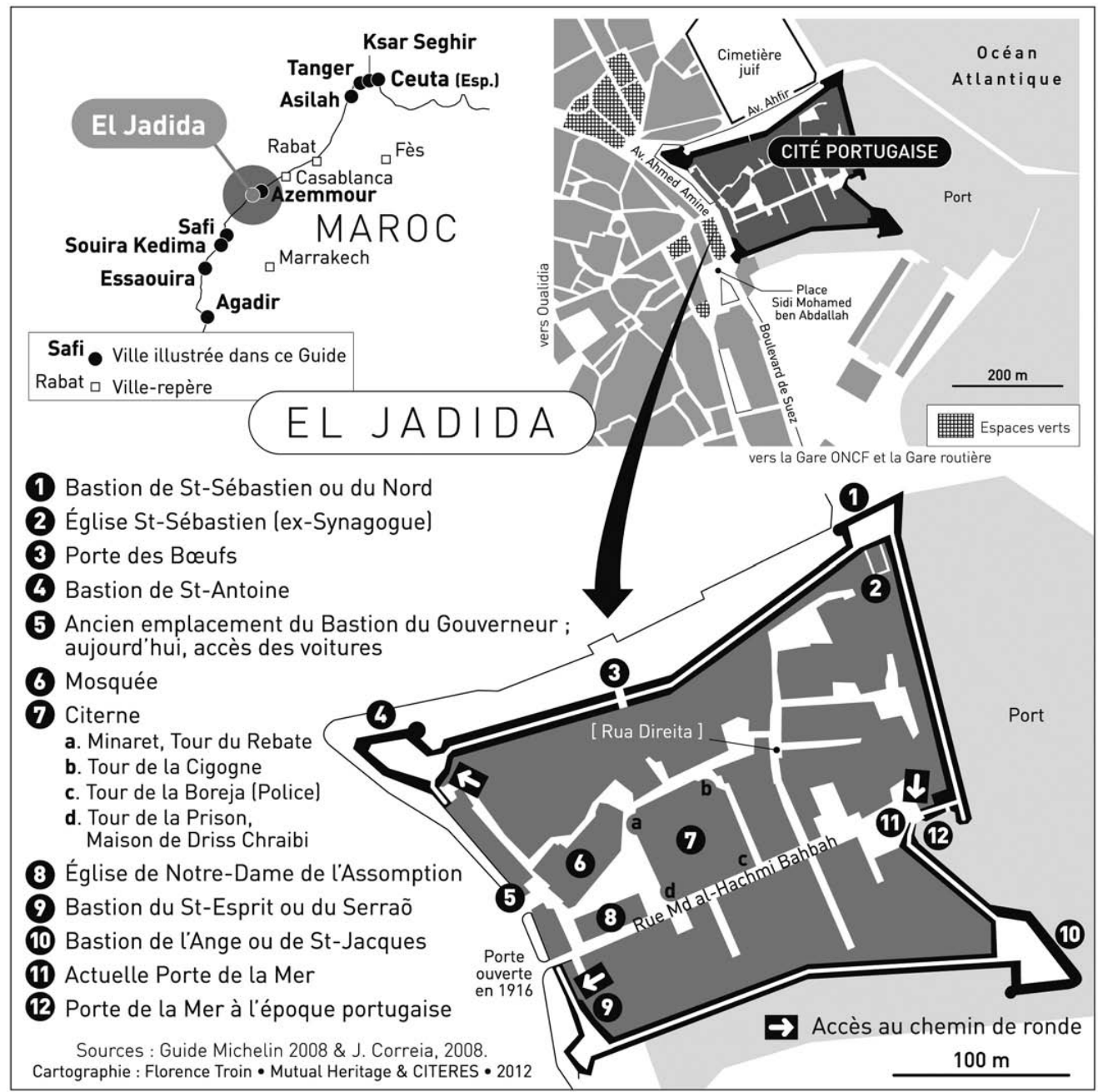

Fig. 2- Descripción de la ciudad. 
Musulmanes, judíos y habitantes de otras nacionalidades cohabitaron dentro del interior de las murallas de la ciudad. La pluralidad racial y religiosa se amplió con la llegada de nuevos europeos, mercaderes, misioneros $\mathrm{y}$ embajadoresLa ciudad se extiende sobre una superficie de unas 7 hectáreas y medio, siendo el fruto de la unión del saber hacer mediterráneo en el arte de la construcción de las fortificaciones. Atestiguando las modificaciones radicales que tuvo la morfología de las fortificaciones entre la época medieval y el Renacimiento. Mazagan, es una fortaleza construida al límite del mar en una bahía natural magnifica, está rodeada de una muralla maciza describiendo una estrella de cuatro brazos que miden cada uno de ellos entre 250 y 300 metros de lado.

Las murallas, ligeramente inclinadas con galibo, tienen una altura aproximada de 8 metros y 10 metros de espesor con el parapeto exterior, que protege el camino de ronda, que tiene 2 metros de ancho.

La fortaleza tiene actualmente cuatro bastiones. El quinto denominado del Gobernador hacia la función de entrada principal, fue destruido por los portugueses en el año 1769 en el momento de abandonar la ciudad.

Los bastiones, y la ciudad son la imagen de la arquitectura de principios de la época moderna caracterizada por el uso de la artillería. Los bastiones eran macizos y muy sólidos para soportar los cañonazos así como los bombardeos de la artillería, constituyendo una punta de flecha (trébol) como prolongación de las murallas. Casamatas y pasillos constituyen la planta baja de los bastiones que se llamaban, El Ángel el del Este, San Sebastián al Norte, San Antonio al Oeste y Santo Espíritu al Sur.

El acceso a estos bastiones se hace mediante tres rampas, una del lado del bastión Santo Espíritu, otra del bastión San Antonio y la tercera en la Puerta del Mar. La fortaleza poseía también tres puertas: la Puerta del Mar en la muralla NordEste que formaba un pequeño puerto, la puerta de los Bueyes en la muralla Nord-Oeste y la puerta principal con un doble arco en medio de la muralla del Sur y que estaba unida a tierra por un puente elevadizo, ya que toda la fortaleza se encontraba rodeada de un foso de unos 20 metros de ancho y 3 metros de profundidad que rellenaban de agua con un sistema de compuertas.

El foso durante el protectorado francés lo rellenaron abriendo incluso una nueva puerta cerca de la puerta principal que daba directamente a la avenida principal llamada Rua da Carreira que constituía el eje principal de la ciudad. En el interior encontramos varios monumentos característicos de esta ciudad.

La iglesia de Nuestra Señora de la Asunción fue una iglesia parroquial construida durante el siglo XVI cuando convirtieron la ciudadela en fortaleza en el año 1541, año que expulsaron a los portugueses de Santa Cruz (Agadir), de Safi y de Azemmour. La iglesia tiene una planta simple, de forma rectangular (44m X 12m) formada por una nave, un coro, una sacristía y su parte trasera. Su techumbre está formada por una armadura de madera con tirantes metálicos, tiene también un campanario cuadrado. En frente de la iglesia se construyo la mezquita ilustrándonos un dialogo de civilizaciones y una continuidad histórica muy respetuosa.

La Cisterna que se encuentra en el centro de la ciudad fue el primer núcleo de esta, era la ciudadela fundada en el año 1514 con una planta rectangular (47m X 56m), rodeada de tres salas en los lados Norte, Este y Sur, flanqueada por cuatro torres: Torre de Cadea al Oeste, Torre de Rebate al Norte, Torre de las Cigüeñas al Este y la Torre El-Brija al Sur, esta es de origen local y fue donde se refugiaron los portugueses en su primera llegada en el año 1502. Las torres de esquina son todas circulares, unidas por unas salas que a su vez encierran una gran sala semisubterranea de $33 \mathrm{~m}$ X $34 \mathrm{~m}$ con 13 columnas y 12 pilares construidos en piedra que soportan un techo nervado de piedra y bóvedas de ladrillo cocido; de estilo gótico manuelino (Rey Manuel $1^{\circ}$ : 1495-1521). Esta gran sala la transformaron en cisterna en el año 1541. En la parte superior de esta se encontraba la guarnición. 


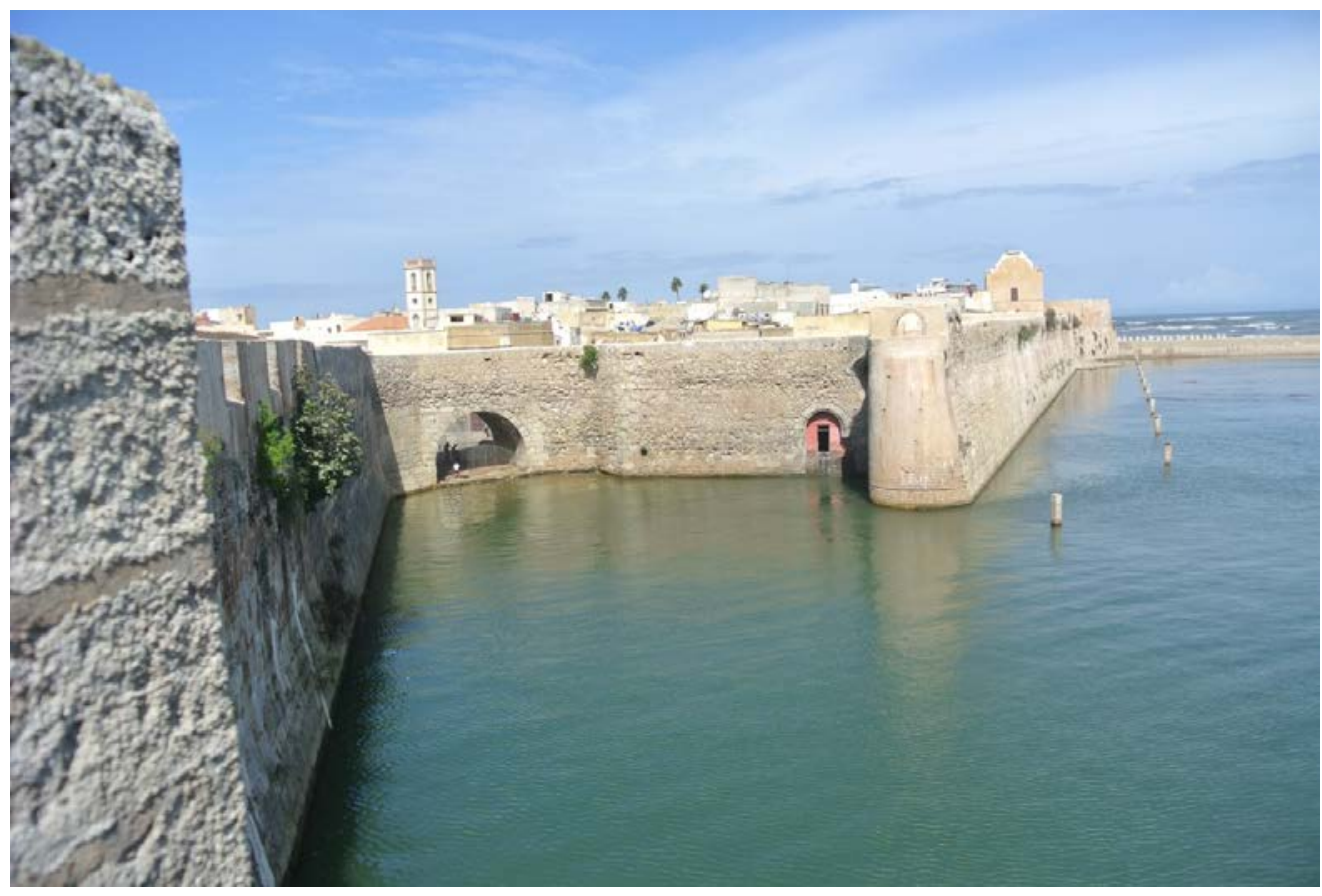

Fig. 3-Murallas. Mazagan.

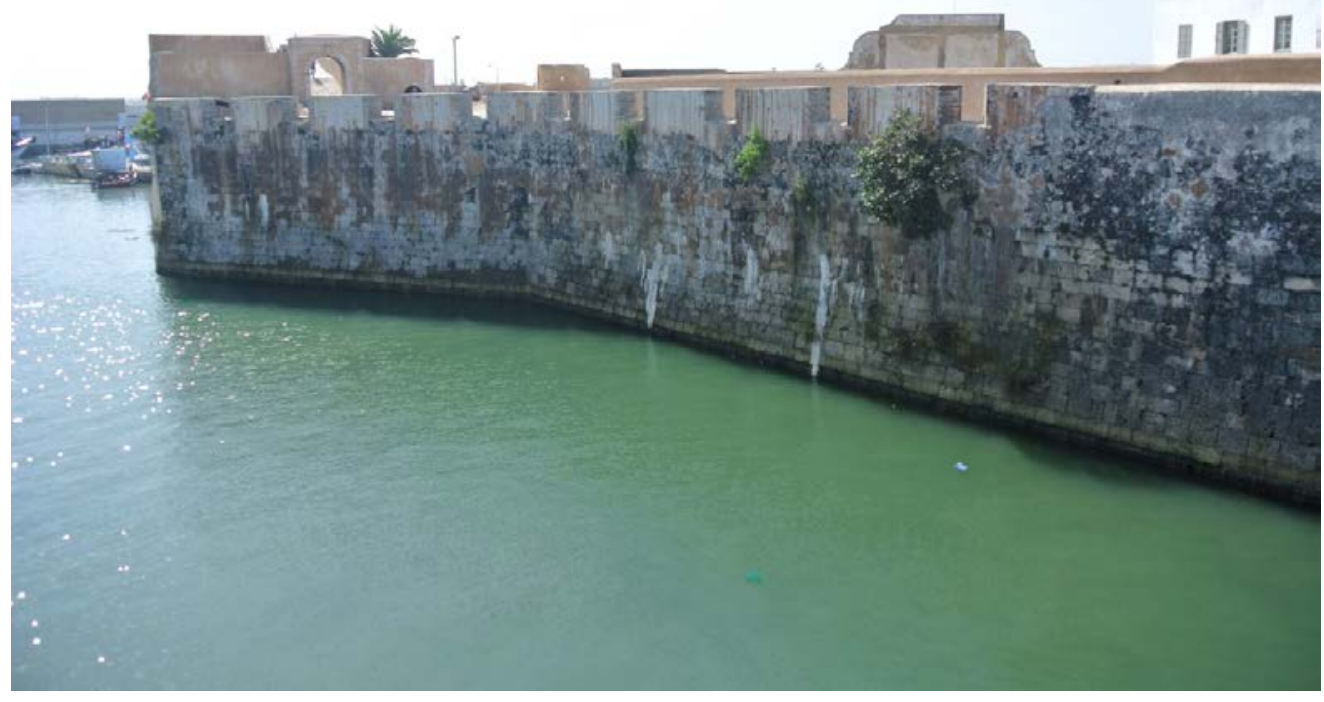

Fig. 4-Murallas. Mazagan. 


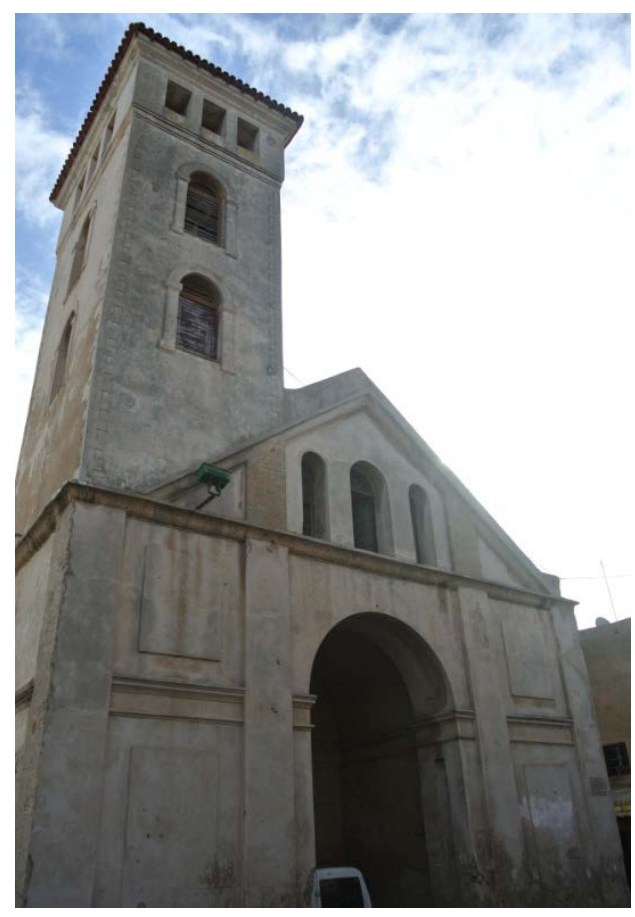

Fig. 5- Iglesia Nuestra Señora de la Asuncion. Mazagan.

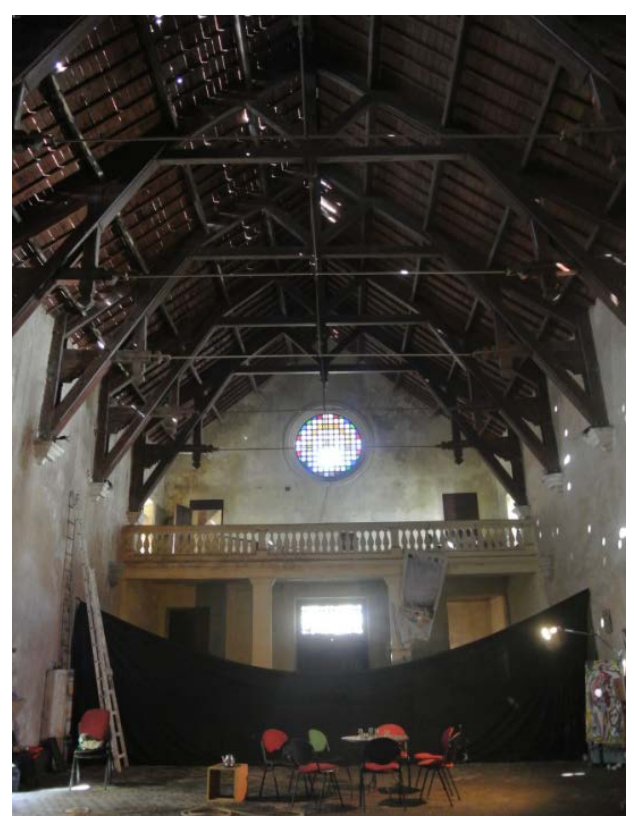

Fig. 6- Iglesia Nuestra Señora de la Asuncion. Mazagan.

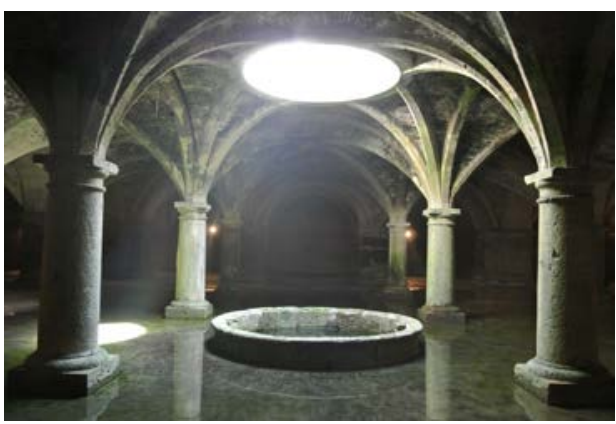

Fig. 7- Cisterna. Mazagan.

\section{Inscripción en la lista de patrimonio mundial en el año 2004 bajo los criterios (II) y (IV)}

\section{Criterio (II)}

La ciudad portuguesa de Mazagan atestigua un intercambio de influencias muy importante entre los siglos XVI y XVIII entre Europa y Marruecos sobre el plano arquitectónico y urbanístico respecto a la planificación de las ciudades. En ella se encuentran monumentos únicos portugueses como:

La cisterna, joya de la arquitectura portuguesa, incomparable en el mundo lusitano, y que supera la pequeña cisterna de Tomar;

El plano de la fortaleza, en forma de estrella con cuatro brazos con las murallas inclinadas en sus puntos medios hacia el interior, representando un modelo único en el mundo lusitano;

Las murallas, por sus bastiones macizos de $10 \mathrm{mts}$. de espesor y $2 \mathrm{mts}$. en el parapeto exterior, no tienen construcciones parecidas ni en la arquitectura portuguesa ni en la arquitectura de las medinas de Marruecos.

El foso, tres años después de construir la fortaleza, los portugueses deciden cavar un foso alrededor de 3mts. de profundidad. Este foso que rodeaba la ciudad por los lados que daban a tierra, permitiendo a la marea alta de llenarlo y así aislar completamente la fortaleza de la tierra. Hoy día queda solamente la parte Sur del foso que se utiliza como dársena naval para la reparación de los barcos de pesca. 


\section{Criterio (IV)}

La ciudad portuguesa de El Jadida, representa una de las etapas sobre la ruta de Indias, ofreciendo un ejemplo eminente de un conjunto arquitectónico que marca el poderío portugués en tierras marroquíes en el momento de los grandes descubrimientos. Representa por este hecho uno de los ejemplos "la arquitectura más espectacular y la mejor preservada militar y citadina del renacimiento, así como de la expansión portuguesa en el mundo”.

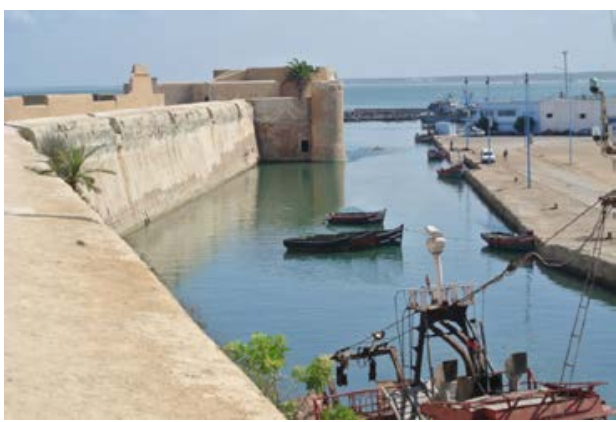

Fig. 8- Foso utilizado como dársena. Mazagan.

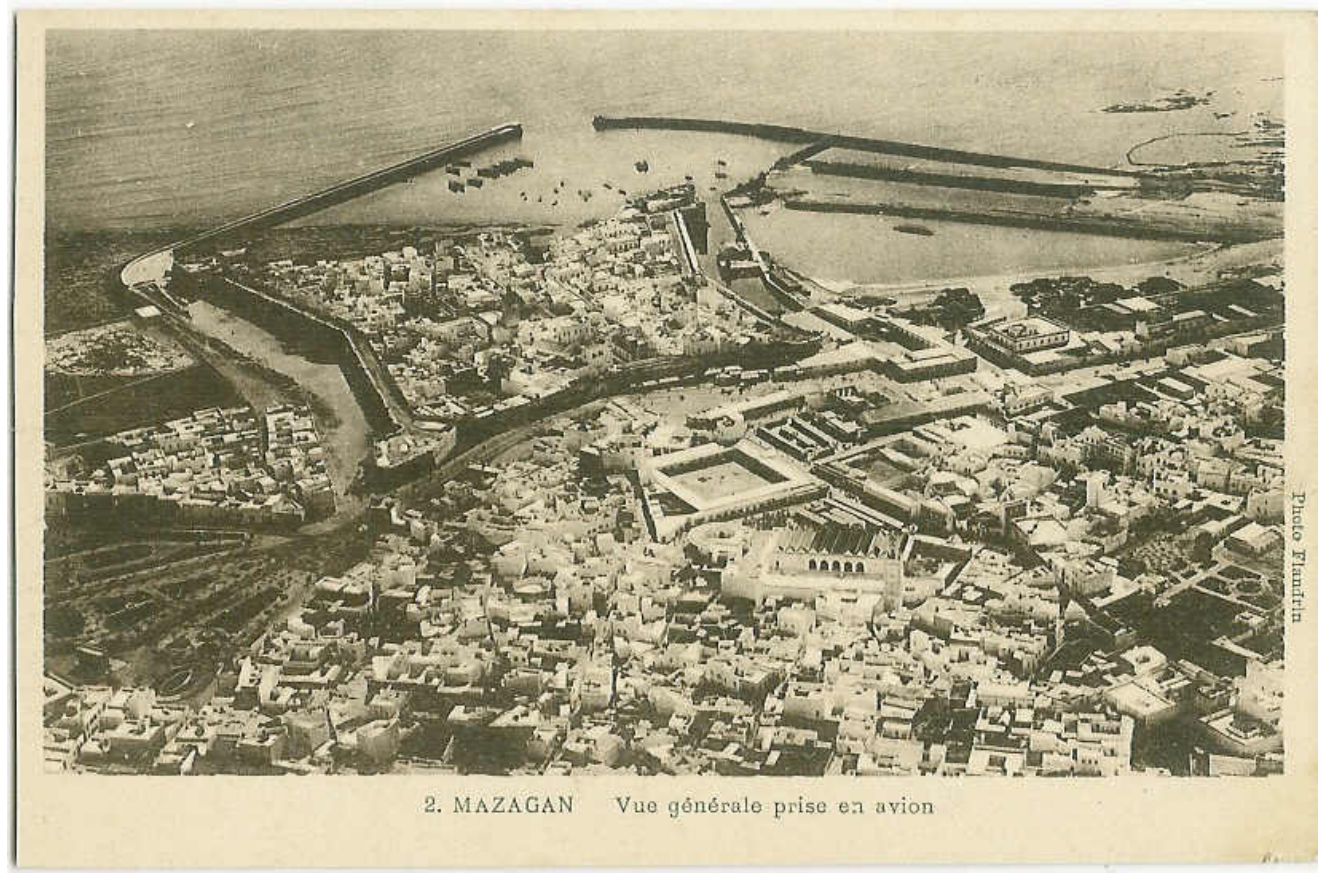

Fig. 9- Antigua fotografía aérea. Mazagan.

\section{Referencias}

Moreira R. (1989) “A arte da portugaise en Afrique. Aperçus sur quelques mythes et certaines réalités“, in Matériaux pour l'histoire de notre temps, n. 32-33. Colonisations en Afrique.

Ricard R. (1940) "Sur la chronologie des fortifications portugaises d'Azemour, Mazagan et Safi“, in III Congresso do Mundo Português - memórias e comunicações, tomo I, vol. III, Lisbonne.

Ricard R. (1932) “ La place de Mazagan : au début du XVIIIème siècle, Paris, Paul Geuthner.

guerra no Renascimento“, in Portugal no mundo: historia das fortificações portuguesas no mundo, Lisbonne, Publicações Alfa.

Moreira R. (1986) "Do rigor teorico à urgencia pratica : a arquitectura militar“, in Historia da arte em Portugal: o limiar do barroco, Lisbonne, Publicações Alfa.

Moreira R. (1981) "A arquitectura militar do renascimento em Portugal“, in A introdução da arte da renascença na península ibérica, Coimbra. 
Mohammed Nadir E., Marinho dos Santos J., (2004), Santa Cruz do Cabo de Guer (Agadir) e a região de sus [policopie] : presença portuguesa (1505-1541), Coimbra, Thèse mestr. Historia da Expansão Portuguesa.

De Oliveira Marques A. (2001) Histoire du Portugal, Paris, Karthala.

Zurfluh J.M. (1994) "Le centre du patrimoine Maroco-lusitanien à El Jadida : Pour une meilleure connaissance des liens historiques entre le Maroc et le Portugal“, in Le Matin Magazine, 7-14 août, pp. 12-13. 